\title{
Stability and Hopf Bifurcation for a Delayed SLBRS Computer Virus Model
}

\author{
Zizhen Zhang ${ }^{1,2}$ and Huizhong Yang ${ }^{2}$ \\ ${ }^{1}$ School of Management Science and Engineering, Anhui University of Finance and Economics, Bengbu 233030, China \\ ${ }^{2}$ Key Laboratory of Advanced Process Control for Light Industry (Ministry of Education), Jiangnan University, Wuxi 214122, China
}

Correspondence should be addressed to Huizhong Yang; yhz@jiangnan.edu.cn

Received 27 June 2014; Accepted 23 July 2014; Published 17 August 2014

Academic Editor: Carlo Bianca

Copyright (c) 2014 Z. Zhang and H. Yang. This is an open access article distributed under the Creative Commons Attribution License, which permits unrestricted use, distribution, and reproduction in any medium, provided the original work is properly cited.

By incorporating the time delay due to the period that computers use antivirus software to clean the virus into the SLBRS model a delayed SLBRS computer virus model is proposed in this paper. The dynamical behaviors which include local stability and Hopf bifurcation are investigated by regarding the delay as bifurcating parameter. Specially, direction and stability of the Hopf bifurcation are derived by applying the normal form method and center manifold theory. Finally, an illustrative example is also presented to testify our analytical results.

\section{Introduction}

For the purpose of studying the prevalence of computer viruses and posing effective strategies of controlling their spread across the Internet and considering the similarity between the propagation of computer virus across the Internet and that of biological viruses across a population, many scholars have proposed computer virus models by properly modifying some epidemiological models [1-6]. In [2], Han and Tan proposed a computer virus model using an SIRS model and investigated the local stability and Hopf bifurcation for the endemic state of the model. In [4], Mishra and Pandey proposed an SEIRS model for the transmission of worms in computer network through vertical transmission and they obtained the threshold value determining whether the worms die out. All the models above assume that an infected computer in which the virus resides is in latency and cannot infect other computers.

Recently, Yang et al. [7] proposed the following SLBRS computer virus model by taking into account the fact that a computer immediately possesses infection ability once it is infected. Consider

$$
\begin{aligned}
& \frac{d S(t)}{d t}=\mu-\beta S(t)(L(t)+B(t))-\mu S(t)+\alpha R(t), \\
& \frac{d L(t)}{d t}=\beta S(t)(L(t)+B(t))-\varepsilon L(t)-\mu L(t), \\
& \frac{d B(t)}{d t}=\varepsilon L(t)-\gamma B(t)-\mu B(t), \\
& \frac{d R(t)}{d t}=\gamma B(t)-\alpha R(t)-\mu R(t),
\end{aligned}
$$

where $S(t)$ denotes the percentage of computers that have no immunity at time $t ; L(t)$ denotes the percentage of infected computers that are latent at time $t ; B(t)$ denotes the percentage of infected computers that are breaking out at time $t$; and $R(t)$ denotes the percentage of uninfected computers that have temporary immunity at time $t . \mu$ is the constant rate for the external computers connecting to the Internet and that for the internal computers disconnecting from the Internet. $\beta$ is the rate of contact for the virus-free computer. $\alpha$ is the rate coefficient from class $R$ to class $S, \varepsilon$ is the rate coefficient from class $L$ to class $B$, and $\gamma$ is the rate coefficient from class $B$ to 
class $R$. Yang et al. [7] studied global stability of the model by constructing a Lyapunov function for the model.

It is well known that there may be a time lag when the computer uses antivirus software to clean the virus and computer virus models with time delay have been studied by many scholars [1, 2, 6, 8-15]. In [8], Feng et al. investigated the Hopf bifurcation of delayed SIRS computer virus propagation model by regarding the latent period of computer virus as a bifurcation parameter. In [10], Liu investigated the Hopf bifurcation of a delayed SEIQRS model for the transmission of malicious objects in computer network by regarding the time delay due to the temporary immunity period as a bifurcation parameter. To the best of our knowledge, few papers deal with the research of Hopf bifurcation of system (1). Motivated by the work above and considering that there may be a time lag when the computer uses antivirus software to clean the virus, we incorporate time delay into system (1) and investigate the Hopf bifurcation of the following delayed SLBRS system:

$$
\begin{aligned}
& \frac{d S(t)}{d t}=\mu-\beta S(t)(L(t)+B(t))-\mu S(t)+\alpha R(t), \\
& \frac{d L(t)}{d t}=\beta S(t)(L(t)+B(t))-\varepsilon L(t)-\mu L(t), \\
& \frac{d B(t)}{d t}=\varepsilon L(t)-\gamma B(t-\tau)-\mu B(t), \\
& \frac{d R(t)}{d t}=\gamma B(t-\tau)-\alpha R(t)-\mu R(t),
\end{aligned}
$$

where $\tau$ is the time delay due to the period that a computer uses antivirus software to clean the virus.

The main purpose of this paper is to investigate the effect of delay on system (2). This paper is organized as follows. In Section 2, sufficient conditions for local stability and existence of local Hopf bifurcation are obtained by analyzing the distribution of the roots of the associated characteristic equation. In Section 3, direction and stability of the Hopf bifurcation are studied by applying the normal form theory and center manifold theorem. In Section 4, some numerical simulations are presented to verify the theoretical results.

\section{Stability and Existence of Local Hopf Bifurcation}

By a simple computation, we can conclude that if $R_{0}>1$, then system (2) has a unique positive equilibrium $D_{*}\left(S_{*}, L_{*}, B_{*}, R_{*}\right)$, where

$$
\begin{aligned}
& S_{*}=\frac{(\varepsilon+\mu)(\gamma+\mu)}{\beta(\varepsilon+\gamma+\mu)}, \\
& L_{*}=\frac{\mu(\alpha+\mu)(\gamma+\mu)\left(1-1 / R_{0}\right)}{(\alpha+\mu)(\varepsilon+\mu)(\gamma+\mu)-\alpha \varepsilon \gamma}, \\
& B_{*}=\frac{\varepsilon L_{*}}{\gamma+\mu}, \quad R_{*}=\frac{\gamma B_{*}}{\alpha+\mu}
\end{aligned}
$$

and $R_{0}=\beta(\varepsilon+\gamma+\mu) /(\varepsilon+\mu)(\gamma+\mu)$ is called the basic reproduction number.
The linearized system of system (2) is

$$
\begin{aligned}
& \frac{d S(t)}{d t}=a_{1} S(t)+a_{2} L(t)+a_{3} B(t)+a_{4} R(t), \\
& \frac{d L(t)}{d t}=a_{5} S(t)+a_{6} L(t)+a_{7} B(t), \\
& \frac{d B(t)}{d t}=a_{8} L(t)+a_{9} B(t)+b_{1} B(t-\tau), \\
& \frac{d R(t)}{d t}=a_{10} R(t)+b_{2} B(t-\tau)
\end{aligned}
$$

where

$$
\begin{gathered}
a_{1}=-\mu-\beta\left(L_{*}+B_{*}\right), \quad a_{2}=-\beta S_{*}, \quad a_{3}=-\beta S_{*}, \\
a_{4}=\alpha, \quad a_{5}=\beta\left(L_{*}+B_{*}\right), \quad a_{6}=\beta S_{*}-\varepsilon-\mu, \\
a_{7}=\beta S_{*}, \quad a_{8}=\varepsilon, \quad a_{9}=-\mu, \\
a_{10}=-(\alpha+\mu), \quad b_{1}=-\gamma, \quad b_{2}=\gamma .
\end{gathered}
$$

The characteristic equation of system (4) is

$$
\begin{aligned}
\lambda^{4}+ & A_{3} \lambda^{3}+A_{2} \lambda^{2}+A_{1} \lambda+A_{0} \\
& +\left(B_{3} \lambda^{3}+B_{2} \lambda^{2}+B_{1} \lambda+B_{0}\right) e^{-\lambda \tau}=0
\end{aligned}
$$

where

$$
\begin{aligned}
A_{0}= & a_{1} a_{6} a_{9} a_{10}+a_{8} a_{10}\left(a_{3} a_{5}-a_{1} a_{7}\right), \\
A_{1}= & a_{7} a_{8} a_{10}+a_{8}\left(a_{1} a_{7}-a_{3} a_{5}\right) \\
& -a_{1} a_{6}\left(a_{9}+a_{10}\right)-a_{9} a_{10}\left(a_{1}+a_{6}\right), \\
A_{2}= & a_{1} a_{6}+a_{9} a_{10}-a_{7} a_{8}+\left(a_{1}+a_{6}\right)\left(a_{9}+a_{10}\right), \\
A_{3}= & -\left(a_{1}+a_{6}+a_{9}+a_{10}\right), \\
B_{0}= & a_{10} b_{1}\left(a_{1} a_{6}-a_{2} a_{5}\right), \\
B_{1}= & \left(a_{2} a_{5}-a_{1} a_{6}-a_{1} a_{10}-a_{6} a_{10}\right) b_{1}, \\
B_{2}= & \left(a_{1}+a_{6}+a_{10}\right) b_{1}, \\
B_{3}= & -b_{1} .
\end{aligned}
$$

For $\tau=0$, (6) reduces to

$$
\lambda^{4}+A_{13} \lambda^{3}+A_{12} \lambda^{2}+A_{11} \lambda+A_{10}=0
$$

where

$$
\begin{array}{ll}
A_{10}=A_{0}+B_{0}, & A_{11}=A_{1}+B_{1}, \\
A_{12}=A_{2}+B_{2}, & A_{13}=A_{3}+B_{3} .
\end{array}
$$


Obviously, if condition $\left(H_{1}\right)$ (10) holds, $D_{*}$ is locally asymptotically stable in the absence of delay according to the Routh-Hurwitz criterion. One has

$$
\begin{aligned}
\operatorname{Det}_{1} & =A_{13}>0, \\
\operatorname{Det}_{2} & =\left|\begin{array}{cc}
A_{13} & 1 \\
A_{11} & A_{12}
\end{array}\right|>0, \\
\operatorname{Det}_{3} & =\left|\begin{array}{ccc}
A_{13} & 1 & 0 \\
A_{11} & A_{12} & A_{13} \\
0 & A_{10} & A_{11}
\end{array}\right|>0, \\
\operatorname{Det}_{4} & =\left|\begin{array}{cccc}
A_{13} & 1 & 0 & 0 \\
A_{11} & A_{12} & A_{13} & 1 \\
0 & A_{10} & A_{11} & A_{12} \\
0 & 0 & 0 & A_{10}
\end{array}\right|>0 .
\end{aligned}
$$

For $\tau>0$, let $\lambda=i \omega(\omega>0)$ be the root of (6). Then, we get

$$
\begin{aligned}
& \left(B_{1} \omega-B_{3} \omega^{3}\right) \sin \tau \omega+\left(B_{0}-B_{2} \omega^{2}\right) \cos \tau \omega \\
& \quad=A_{2} \omega^{2}-\omega^{4}-A_{0}, \\
& \left(B_{1} \omega-B_{3} \omega^{3}\right) \cos \tau \omega-\left(B_{0}-B_{2} \omega^{2}\right) \sin \tau \omega \\
& =A_{3} \omega^{3}-A_{1} \omega,
\end{aligned}
$$

from which one can get

$$
\omega^{8}+c_{3} \omega^{6}+c_{2} \omega^{4}+c_{1} \omega^{2}+c_{0}=0,
$$

with

$$
\begin{aligned}
& c_{0}=A_{0}^{2}-B_{0}^{2}, \\
& c_{1}=A_{1}^{2}-B_{1}^{2}-2 A_{0} A_{2}+2 B_{0} B_{2}, \\
& c_{2}=A_{2}^{2}-B_{2}^{2}+2 A_{0}-2 A_{1} A_{3}+2 B_{1} B_{3}, \\
& c_{3}=A_{3}^{2}-B_{3}^{2}-2 A_{2} .
\end{aligned}
$$

Let $\omega^{2}=v$; then, (12) becomes

$$
v^{4}+c_{3} v^{3}+c_{2} v^{2}+c_{1} v+c_{0}=0 .
$$

In order to give the main results in this paper, we make the following assumption.

$\left(\mathrm{H}_{2}\right)$ Equation (14) has at least one positive root.

If condition $\left(\mathrm{H}_{2}\right)$ holds, we know that (12) has at least a positive root $\omega_{0}$ such that (6) has a pair of purely imaginary roots $\pm i \omega_{0}$. For $\omega_{0}$, the corresponding critical value of the delay is

$$
\tau_{0}=\frac{1}{\omega_{0}} \arccos \frac{p_{6} \omega_{0}^{6}+p_{4} \omega_{0}^{4}+p_{2} \omega_{0}^{2}+p_{0}}{q_{6} \omega_{0}^{6}+q_{4} \omega_{0}^{4}+q_{2} \omega_{0}^{2}+q_{0}},
$$

where

$$
\begin{gathered}
p_{0}=-A_{0} B_{0}, \quad p_{2}=A_{0} B_{2}-A_{1} B_{1}+A_{2} B_{0}, \\
p_{4}=A_{1} B_{3}-A_{2} B_{2}+A_{3} B_{1}-B_{0}, \\
p_{6}=B_{2}-A_{3} B_{3}, \quad q_{0}=B_{0}^{2}, \quad q_{2}=B_{1}^{2}-2 B_{0} B_{2}, \\
q_{4}=B_{2}^{2}-2 B_{1} B_{3}, \quad q_{6}=B_{3}^{2} .
\end{gathered}
$$

Taking the derivative with respect to $\tau$ on both sides of (6), we obtain

$$
\begin{aligned}
{\left[\frac{d \lambda}{d \tau}\right]^{-1}=} & -\frac{4 \lambda^{3}+3 A_{3} \lambda^{2}+2 A_{2} \lambda+A_{1}}{\lambda\left(\lambda^{4}+A_{3} \lambda^{3}+A_{2} \lambda^{2}+A_{1} \lambda+A_{0}\right)} \\
& +\frac{3 B_{3} \lambda^{2}+2 B_{2} \lambda+B_{1}}{\lambda\left(B_{3} \lambda^{3}+B_{2} \lambda^{2}+B_{1} \lambda+B_{0}\right)} .
\end{aligned}
$$

Thus,

$$
\begin{aligned}
\operatorname{Re} & {\left[\frac{d \lambda}{d \tau}\right]_{\tau=\tau_{0}}^{-1} } \\
& =\frac{f^{\prime}\left(v_{*}\right)}{B_{3}^{2} \omega_{0}^{6}+\left(B_{2}^{2}-2 B_{1} B_{3}\right) \omega_{0}^{4}+\left(B_{1}^{2}-2 B_{0} B_{2}\right) \omega_{0}^{2}+B_{0}^{2}},
\end{aligned}
$$

where $v_{*}=\omega_{0}^{2}$ and $f(v)=v^{4}+c_{3} v^{3}+c_{2} v^{2}+c_{1} v+c_{0}$.

Thus, if condition $\left(H_{3}\right) f^{\prime}\left(v_{*}\right) \neq 0$, then $\operatorname{Re}[d \lambda / d \tau]_{\tau=\tau_{0}}^{-1} \neq$ 0 . According to the Hopf bifurcation theorem in [16], we have the following results.

Theorem 1. If conditions $\left(\mathrm{H}_{1}\right)-\left(\mathrm{H}_{3}\right)$ hold, then the positive equilibrium $D_{*}\left(S_{*}, L_{*}, B_{*}, R_{*}\right)$ of system (2) is asymptotically stable for $\tau \in\left[0, \tau_{0}\right)$ and system (2) undergoes a Hopf bifurcation at the positive equilibrium $D_{*}\left(S_{*}, L_{*}, B_{*}, R_{*}\right)$ when $\tau=$ $\tau_{0}$.

\section{Properties of the Hopf Bifurcation}

Let $u_{1}(t)=S(t)-S_{*}, u_{2}(t)=L(t)-L_{*}, u_{3}(t)=B(t)-B_{*}$, $u_{4}(t)=R(t)-R_{*}$, and $\tau=\tau_{0}+\mu, \mu \in R$, and normalize the delay by $t \rightarrow(t / \tau)$. Then, system (2) can be transformed into a functional differential equation (PDF) as

$$
\dot{u}(t)=L_{\mu} u_{t}+F\left(\mu, u_{t}\right),
$$

where

$$
\begin{aligned}
\begin{aligned}
u_{t} & =\left(u_{1}(t), u_{2}(t), u_{3}(t), u_{4}(t)\right)^{T} \in C \\
& =C\left([-1,0], R^{4}\right), \\
L_{\mu} \phi & =\left(\tau_{0}+\mu\right)\left(A^{\prime} \phi(0)+B^{\prime} \phi(-1)\right), \\
F\left(\mu, u_{t}\right) & \\
& =\left(\tau_{0}+\mu\right)\left(\begin{array}{c}
-\beta\left(\phi_{1}(0) \phi_{2}(0)+\phi_{1}(0) \phi_{3}(0)\right) \\
\beta\left(\phi_{1}(0) \phi_{2}(0)+\phi_{1}(0) \phi_{3}(0)\right) \\
0 \\
0
\end{array}\right),
\end{aligned}
\end{aligned}
$$

where

$$
A^{\prime}=\left(\begin{array}{cccc}
a_{1} & a_{2} & a_{3} & a_{4} \\
a_{5} & a_{6} & a_{7} & 0 \\
0 & a_{8} & a_{9} & 0 \\
0 & 0 & 0 & a_{10}
\end{array}\right), \quad B^{\prime}=\left(\begin{array}{cccc}
0 & 0 & 0 & 0 \\
0 & 0 & 0 & 0 \\
0 & 0 & b_{1} & 0 \\
0 & 0 & b_{2} & 0
\end{array}\right) \text {. }
$$


By the Riesz representation theorem, there is a $4 \times 4$ matrix function with bounded variation components $\eta(\theta, \mu)$, $\theta \in[-1,0]$ such that

$$
L_{\mu} \phi=\int_{-1}^{0} d \eta(\theta, \mu) \phi(\theta), \quad \phi \in C .
$$

In fact, we choose

$$
\eta(\theta, \mu)=\left(\tau_{0}+\mu\right)\left(A^{\prime} \delta(\theta)+B^{\prime} \delta(\theta+1)\right) .
$$

For $\left.\phi \in C([-1,0]), R^{4}\right)$, we define

$$
\begin{aligned}
& A(\mu) \phi= \begin{cases}\frac{d \phi(\theta)}{d \theta}, & -1 \leq \theta<0, \\
\int_{-1}^{0} d \eta(\theta, \mu) \phi(\theta), & \theta=0,\end{cases} \\
& R(\mu) \phi= \begin{cases}0, & -1 \leq \theta<0, \\
F(\mu, \phi), & \theta=0 .\end{cases}
\end{aligned}
$$

Then, system (19) is equivalent to the following form:

$$
\dot{u}(t)=A(\mu) u_{t}+R(\mu) u_{t} .
$$

Next, we define the adjoint operator $A^{*}$ of $A$ as

$$
A^{*}(\mu) s= \begin{cases}-\frac{d \varphi(s)}{d s}, & 0<s \leq 1, \\ \int_{-1}^{0} d \eta^{T}(s, \mu) \varphi(-s), & s=0\end{cases}
$$

and a bilinear inner product as

$$
\langle\varphi, \phi\rangle=\bar{\varphi}(0) \phi(0)-\int_{\theta=-1}^{0} \int_{\xi=0}^{\theta} \bar{\varphi}(\xi-\theta) d \eta(\theta) \phi(\xi) d \xi,
$$

where $\eta(\theta)=\eta(\theta, 0)$.

Let $\rho(\theta)=\left(1, \rho_{2}, \rho_{3}, \rho_{4}\right)^{T} e^{i \tau_{0} \omega_{0} \theta}$ be the eigenvector of $A(0)$ corresponding to the eigenvalue $+i \tau_{0} \omega_{0}$ and let $\rho^{*}(\theta)=D\left(1, \rho_{2}^{*}, \rho_{3}^{*}, \rho_{4}^{*}\right) e^{i \tau_{0} \omega_{0} s}$ be the eigenvector of $A^{*}(0)$ corresponding to the eigenvalue $-i \tau_{0} \omega_{0}$. Then, from the definition of $A(0)$ and $A^{*}(0)$, we obtain

$$
\begin{gathered}
\rho_{2}=\frac{a_{5}+a_{7} \rho_{3}}{\tau_{0} \omega_{0}-a_{6}}, \quad \rho_{3}=\frac{a_{5} a_{8}}{\left(i \omega_{0}-a_{6}\right)\left(i \omega_{0}-a_{9}-b_{1} e^{-i \tau_{0} \omega_{0}}\right)}, \\
\rho_{4}=\frac{i \omega_{0}-a_{1}-a_{2} \rho_{2}-a_{3} \rho_{3}}{a_{4}}, \\
\rho_{2}^{*}=-\frac{i \omega_{0}+a_{1}}{a_{5}}, \quad \rho_{3}^{*}=-\frac{a_{2}+\left(i \omega_{0}+a_{6}\right) \rho_{2}^{*}}{a_{8}}, \\
\rho_{4}^{*}=-\frac{a_{3}+a_{7} \rho_{2}^{*}+\left(i \omega_{0}+a_{9}+b_{1} e^{i \tau_{0} \omega_{0}}\right) \rho_{2}^{*}}{b_{2} e^{i \tau_{0} \omega_{0}}} .
\end{gathered}
$$

From (27), we can get

$$
\begin{aligned}
\left\langle q^{*}(s), q(\theta)\right\rangle=\bar{\rho}[ & 1+\rho_{2} \bar{\rho}_{2}^{*}+\rho_{3} \bar{\rho}_{3}^{*}+\rho_{4} \bar{\rho}_{4}^{*} \\
& \left.+\tau_{0} \rho_{3} e^{-i \tau_{0} \omega_{0}}\left(b_{1} \bar{\rho}_{3}^{*}+b_{2} \bar{\rho}_{4}^{*}\right)\right] .
\end{aligned}
$$

Then, we choose

$$
\begin{aligned}
\bar{D}=[1 & +\rho_{2} \bar{\rho}_{2}^{*}+\rho_{3} \bar{\rho}_{3}^{*}+\rho_{4} \bar{\rho}_{4}^{*} \\
& \left.+\tau_{0} \rho_{3} e^{-i \tau_{0} \omega_{0}}\left(b_{1} \bar{\rho}_{3}^{*}+b_{2} \bar{\rho}_{4}^{*}\right)\right]^{-1}
\end{aligned}
$$

such that $\left\langle\rho^{*}, \rho\right\rangle=1$ and $\left\langle\rho^{*}, \bar{\rho}\right\rangle=0$.

Following the algorithms given in [16] and using similar computation process in [17], we can get the following important coefficients:

$$
\begin{aligned}
& g_{20}=2 \tau_{0} \bar{D} \beta\left(\bar{\rho}_{2}^{*} \rho_{3}-\rho_{2}\right), \\
& g_{11}=\tau_{0} \bar{D} \beta\left(\bar{\rho}_{2}^{*}\left(\rho_{3}+\bar{\rho}_{3}\right)-\left(\rho_{2}+\bar{\rho}_{2}\right)\right), \\
& g_{02}=2 \tau_{0} \bar{D} \beta\left(\bar{\rho}_{2}^{*} \bar{\rho}_{3}-\bar{\rho}_{2}\right), \\
& g_{21}=2 \tau_{0} \bar{D} \beta\left(\overline { \rho } _ { 2 } ^ { * } \left(W_{11}^{(1)}(0) \rho_{3}+\frac{1}{2} W_{20}^{(1)}(0) \bar{\rho}_{3}\right.\right. \\
& \left.+W_{11}^{(3)}(0)+\frac{1}{2} W_{20}^{(3)}\right) \\
& -\left(W_{11}^{(1)}(0) \rho_{2}+\frac{1}{2} W_{20}^{(1)}(0) \bar{\rho}_{2}\right. \\
& \left.\left.\quad+W_{11}^{(2)}(0)+\frac{1}{2} W_{20}^{(2)}\right)\right),
\end{aligned}
$$

with

$$
\begin{aligned}
& W_{20}(\theta)=\frac{i g_{20} \rho(0)}{\tau_{0} \omega_{0}} e^{i \tau_{0} \omega_{0} \theta}+\frac{i \bar{g}_{02} \bar{\rho}(0)}{3 \tau_{0} \omega_{0}} e^{-i \tau_{0} \omega_{0} \theta}+E_{1} e^{2 i \tau_{0} \omega_{0} \theta}, \\
& W_{11}(\theta)=-\frac{i g_{11} \rho(0)}{\tau_{0} \omega_{0}} e^{i \tau_{0} \omega_{0} \theta}+\frac{i \bar{g}_{11} \bar{\rho}(0)}{\tau_{0} \omega_{0}} e^{-i \tau_{0} \omega_{0} \theta}+E_{2},
\end{aligned}
$$

where $E_{1}$ and $E_{2}$ can be determined by the following equations, respectively,

$$
\begin{gathered}
\left(\begin{array}{cccc}
2 i \omega_{0}-a_{1} & -a_{2} & -a_{3} & -a_{4} \\
-a_{5} & 2 i \omega_{0}-a_{6} & -a_{7} & 0 \\
0 & -a_{8} & 2 i \omega_{0}-a_{9}-b_{1} e^{-2 i \tau_{0} \omega_{0}} & 0 \\
0 & 0 & -b_{2} e^{-2 i \tau_{0} \omega_{0}} & 2 i \omega_{0}-a_{10}
\end{array}\right) \\
=2\left(\begin{array}{c}
E_{1}^{(1)} \\
E_{1}^{(2)} \\
0 \\
0
\end{array}\right), \\
\left(\begin{array}{cccc}
a_{1} & a_{2} & a_{3} & a_{4} \\
a_{5} & a_{6} & a_{7} & 0 \\
0 & a_{8} & a_{9}+b_{1} & 0 \\
0 & 0 & b_{2} & a_{10}
\end{array}\right) E_{2}=-\left(\begin{array}{c}
E_{2}^{(1)} \\
E_{2}^{(2)} \\
0 \\
0
\end{array}\right),
\end{gathered}
$$

with

$$
\begin{array}{cl}
E_{1}^{(1)}=-\beta \rho_{2}, & E_{1}^{(2)}=\beta \rho_{2}, \\
E_{2}^{(1)}=-\beta\left(\rho_{2}+\bar{\rho}_{2}\right), & E_{2}^{(2)}=\beta\left(\rho_{3}+\bar{\rho}_{3}\right) .
\end{array}
$$


Then, we can get the following coefficients:

$$
\begin{aligned}
C_{1}(0) & =\frac{i}{2 \tau_{0} \omega_{0}}\left(g_{11} g_{20}-2\left|g_{11}\right|^{2}-\frac{\left|g_{02}\right|^{2}}{3}\right)+\frac{g_{21}}{2}, \\
\mu_{2} & =-\frac{\operatorname{Re}\left\{C_{1}(0)\right\}}{\operatorname{Re}\left\{\lambda^{\prime}\left(\tau_{0}\right)\right\}} \\
\beta_{2} & =2 \operatorname{Re}\left\{C_{1}(0)\right\}, \\
T_{2} & =-\frac{\operatorname{Im}\left\{C_{1}(0)\right\}+\mu_{2} \operatorname{Im}\left\{\lambda^{\prime}\left(\tau_{0}\right)\right\}}{\tau_{0} \omega_{0}} .
\end{aligned}
$$

In conclusion, we have the following results.

Theorem 2. For system (2), If $\mu_{2}>0\left(\mu_{2}<0\right)$, then the Hopf bifurcation is supercritical (subcritical). If $\beta_{2}<0\left(\beta_{2}>0\right)$, then the bifurcating periodic solutions are stable (unstable). If $T_{2}>0\left(T_{2}<0\right)$, then the period of the bifurcating periodic solutions increases (decreases).

\section{Numerical Simulation}

In this section, we present a numerical example to justify the theoretical results above. We use the same coefficients as those used in [7] and consider the following system:

$$
\begin{aligned}
\frac{d S(t)}{d t}= & 0.001-0.15 S(t)(L(t)+B(t)) \\
& -0.001 S(t)+0.01 R(t), \\
\frac{d L(t)}{d t}= & 0.15 S(t)(L(t)+B(t))-0.05 L(t)-0.001 L(t), \\
\frac{d B(t)}{d t}= & 0.05 L(t)-0.02 B(t-\tau)-0.001 B(t), \\
\frac{d R(t)}{d t}= & 0.02 B(t-\tau)-0.01 R(t)-0.001 R(t),
\end{aligned}
$$

from which we obtain the basic reproduction number $R_{0}=9.944>1$ and the unique positive equilibrium $D_{*}(0.1006,0.1160,0.2762,0.5022)$ of system (36). Further, we obtain $\omega_{0}=2.3091, \tau_{0}=58.5472$. According to Theorem 1 in Section 2, we know that, when $\tau \in[0,58.5472)$, the positive equilibrium $D_{*}(0.1006,0.1160,0.2762,0.5022)$ of system (36) is locally asymptotically stable. As can be seen from Figures 1 and 2 , when $\tau=55.2386<\tau_{0}$, the positive equilibrium $D_{*}(0.1006,0.1160,0.2762,0.5022)$ of system (36) is locally asymptotically stable. However, when the delay passes through the critical value of $\tau_{0}=58.5472$, the positive equilibrium $D_{*}$ will lose stability and a Hopf bifurcation occurs. This property can be illustrated by Figures 3 and 4 .

In addition, by some complex computations and from (35), we obtain $\mu_{2}=-0.8143<0, \beta_{2}=-0.9001<0$, and $T_{2}=0.0466>0$. Therefore, we can conclude that the Hopf bifurcation of system (36) is subcritical, the bifurcating periodic solutions are stable, and the period of the periodic solutions decreases according to Theorem 2 in Section 3.

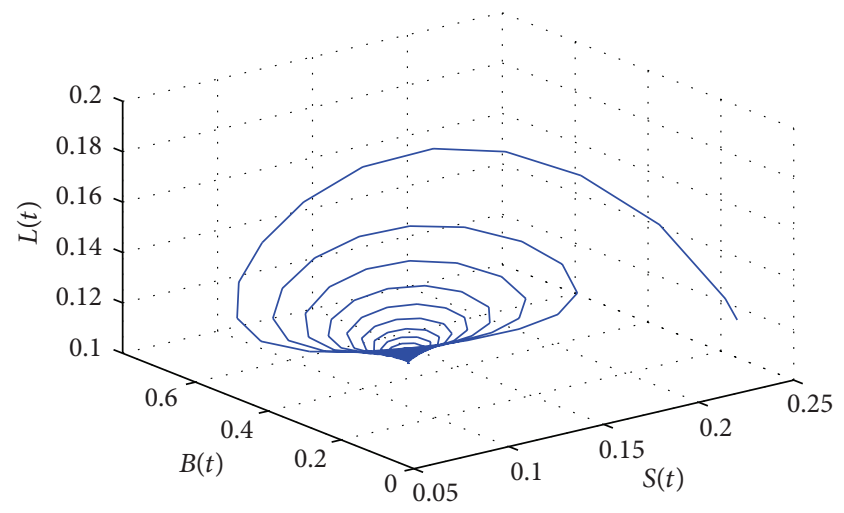

Figure 1: The phase plot of states $S, L$, and $B$ for $\tau=55.2386<\tau_{0}=$ 58.5472 .

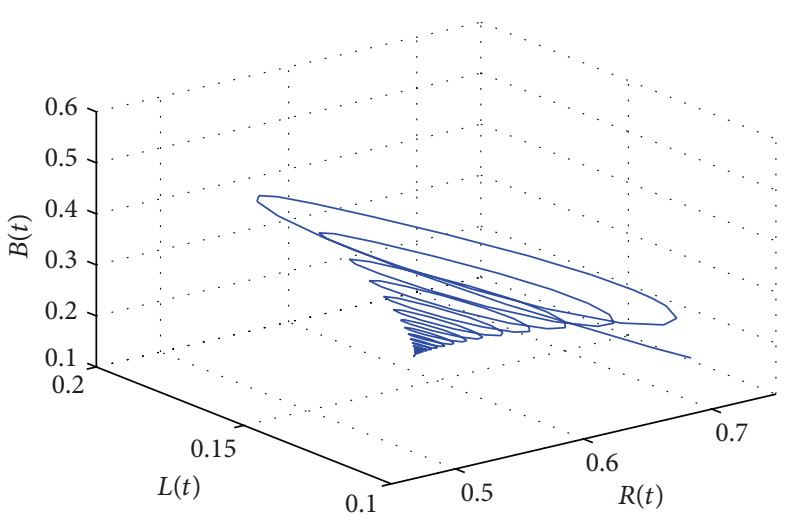

FIGURE 2: The phase plot of states $L, B$, and $R$ for $\tau=55.2386<\tau_{0}=$ 58.5472 .

\section{Conclusions}

A delayed SLBRS computer virus model has been studied in this paper based on the model proposed in [7]. Compared with literature [7], we mainly consider the effect of the time delay due to the period that the computers use antivirus software to clean the virus on the model. The main results are presented in terms of local stability and local Hopf bifurcation. Sufficient conditions for local stability and existence of local Hopf bifurcation are obtained by analyzing the distribution of the roots of the associated characteristic equation. It has been shown that when the delay is suitably small $\left(\tau<\tau_{0}\right)$, the computer virus model is locally asymptotically stable. In such case, the propagation of the computer virus can be easily controlled. However, once the delay passes though the critical value $\tau_{0}$, a Hopf bifurcation occurs and a family of periodic solutions bifurcates from the positive equilibrium of the model. This is not welcome in networks because the computer virus will be out of control in this case. Further, direction and stability of the Hopf bifurcation have also been determined by using the normal form method and center manifold theory. Numerical simulations are also included to verify the analytical predictions. 


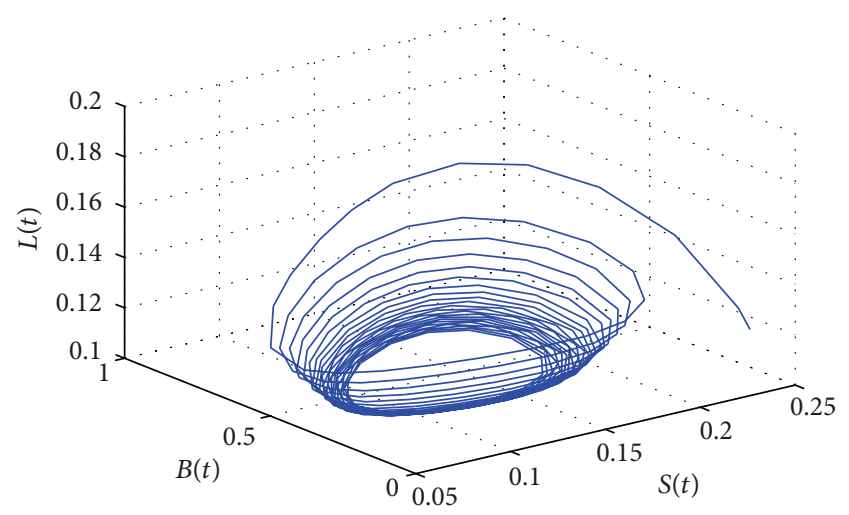

FIGURE 3: The phase plot of states $S, L$, and $B$ for $\tau=60.5386>\tau_{0}=$ 58.5472 .

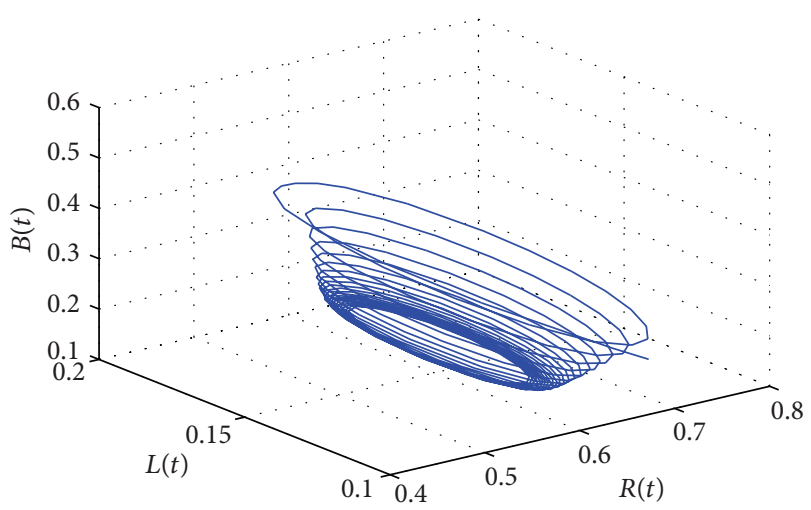

FIGURE 4: The phase plot of states $L, B$, and $R$ for $\tau=60.5386>\tau_{0}=$ 58.5472 .

\section{Conflict of Interests}

The authors declare that there is no conflict of interests regarding the publication of this paper.

\section{Acknowledgments}

This work was supported by the National Nature Science Foundation of China (61273070), a project funded by the Priority Academic Program Development of Jiangsu Higher Education Institutions, and Natural Science Foundation of the Higher Education Institutions of Anhui Province (KJ2014A005).

\section{References}

[1] B. K. Mishra and D. K. Saini, "SEIRS epidemic model with delay for transmission of malicious objects in computer network," Applied Mathematics and Computation, vol. 188, no. 2, pp. 14761482, 2007.

[2] X. Han and Q. L. Tan, "Dynamical behavior of computer virus on Internet," Applied Mathematics and Computation, vol. 217, no. 6, pp. 2520-2526, 2010.

[3] B. K. Mishra and S. K. Pandey, "Fuzzy epidemic model for the transmission of worms in computer network," Nonlinear
Analysis: Real World Applications, vol. 11, no. 5, pp. 4335-4341, 2010.

[4] B. K. Mishra and S. K. Pandey, "Dynamic model of worms with vertical transmission in computer network," Applied Mathematics and Computation, vol. 217, no. 21, pp. 8438-8446, 2011.

[5] B. K. Mishra and N. Jha, "SEIQRS model for the transmission of malicious objects in computer network," Applied Mathematical Modelling, vol. 34, no. 3, pp. 710-715, 2010.

[6] J. Ren, X. Yang, L.-X. Yang, Y. Xu, and F. Yang, "A delayed computer virus propagation model and its dynamics," Chaos, Solitons \& Fractals, vol. 45, no. 1, pp. 74-79, 2012.

[7] M. Yang, Z. Zhang, Q. Li, and G. Zhang, "An SLBRS model with vertical transmission of computer virus over the Internet," Discrete Dynamics in Nature and Society, vol. 2012, Article ID 925648, 17 pages, 2012.

[8] L. Feng, X. Liao, H. Li, and Q. Han, "Hopf bifurcation analysis of a delayed viral infection model in computer networks," Mathematical and Computer Modelling, vol. 56, no. 7-8, pp. 167179, 2012.

[9] Z. Z. Zhang and H. Z. Yang, "Hopf bifurcation analysis for a computer virus model with two delays," Abstract and Applied Analysis, vol. 2013, Article ID 560804, 18 pages, 2013.

[10] J. Liu, "Hopf bifurcation in a delayed SEIQRS model for the transmission of malicious objects in computer network," Journal of Applied Mathematics, vol. 2014, Article ID 492198, 8 pages, 2014.

[11] Y. Yao, W. L. Xiang, A. D. Qu, G. Yu, and F. X. Gao, "Hopf bifurcation in an SEIDQV worm propagation model with quarantine strategy," Discrete Dynamics in Nature and Society, vol. 2012, Article ID 304868, 18 pages, 2012.

[12] T. Dong, X. Liao, and H. Li, "Stability and Hopf bifurcation in a computer virus model with multistate antivirus," Abstract and Applied Analysis, vol. 2012, Article ID 841987, 16 pages, 2012.

[13] Y. Yao, X. Xie, H. Guo, G. Yu, F. Gao, and X. Tong, "Hopf bifurcation in an internet worm propagation model with time delay in quarantine," Mathematical and Computer Modelling, vol. 57, no. 11-12, pp. 2635-2646, 2013.

[14] S. Wang, Q. Liu, X. Yu, and Y. Ma, "Bifurcation analysis of a model for network worm propagation with time delay," Mathematical and Computer Modelling, vol. 52, no. 3-4, pp. 435447, 2010.

[15] Y. Yao, N. Zhang, W. Xiang, G. Yu, and F. Gao, "Modeling and analysis of bifurcation in a delayed worm propagation model," Journal of Applied Mathematics, vol. 2013, Article ID 927369, 11 pages, 2013.

[16] B. D. Hassard, N. D. Kazarinoff, and Y. H. Wan, Theory and Applications of Hopf Bifurcation, Cambridge University Press, Cambridge, UK, 1981.

[17] C. Bianca, M. Ferrara, and L. Guerrini, "The Cai model with time delay: existence of periodic solutions and asymptotic analysis," Applied Mathematics \& Information Sciences, vol. 7, no. 1, pp. 21-27, 2013. 


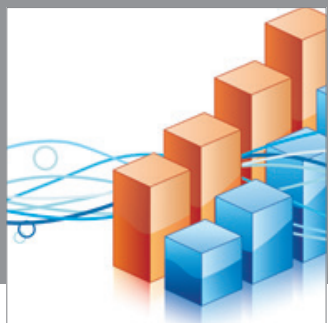

Advances in

Operations Research

mansans

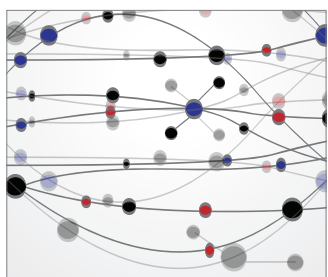

The Scientific World Journal
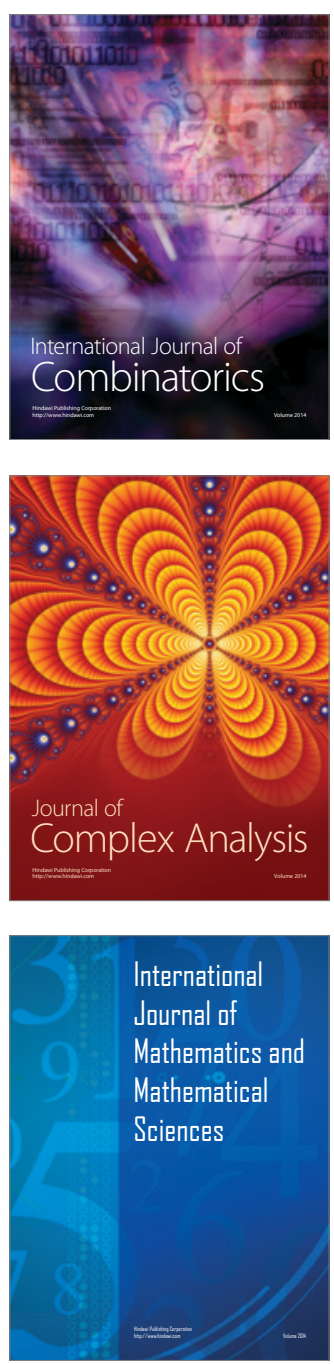
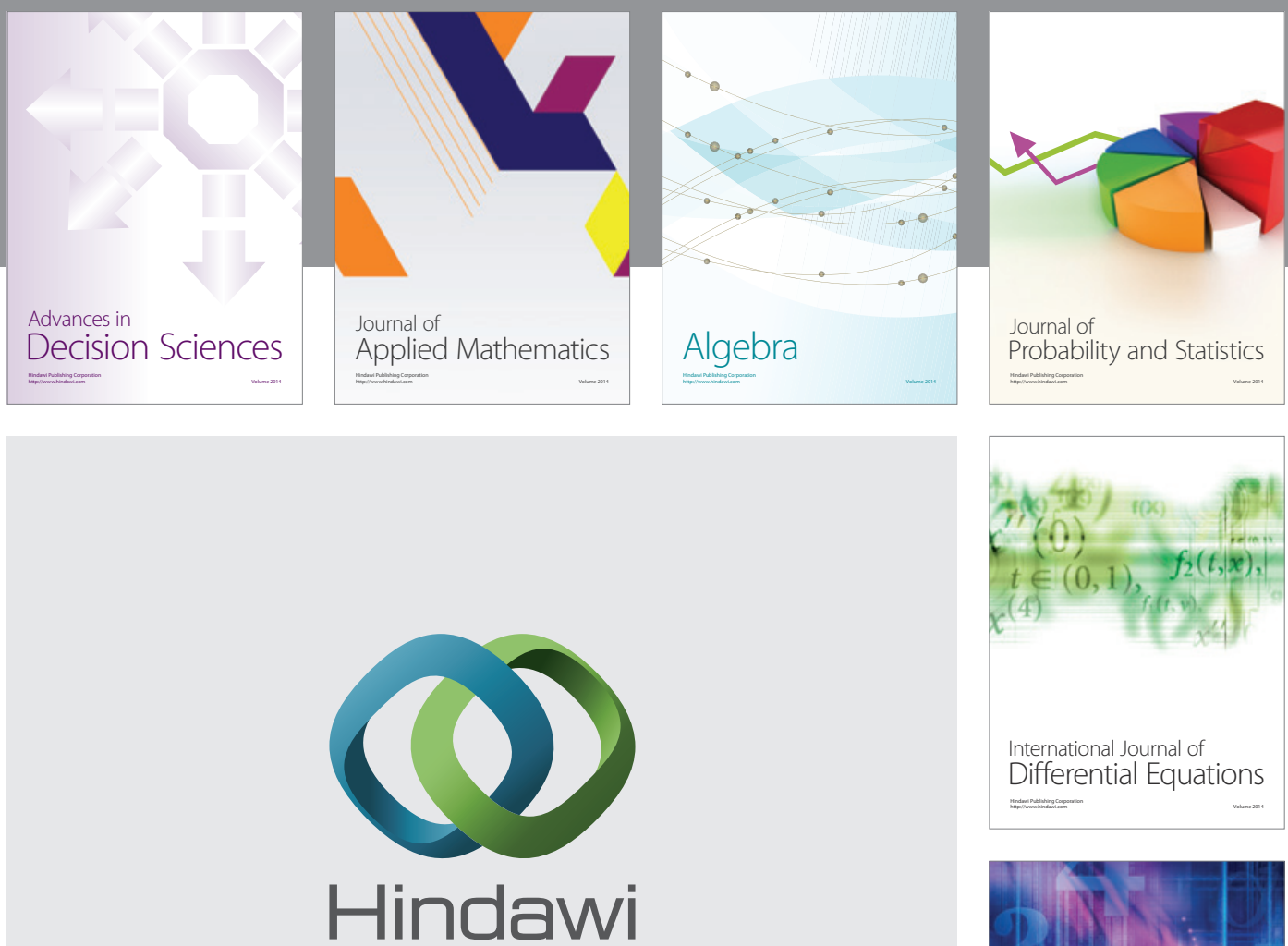

Submit your manuscripts at http://www.hindawi.com
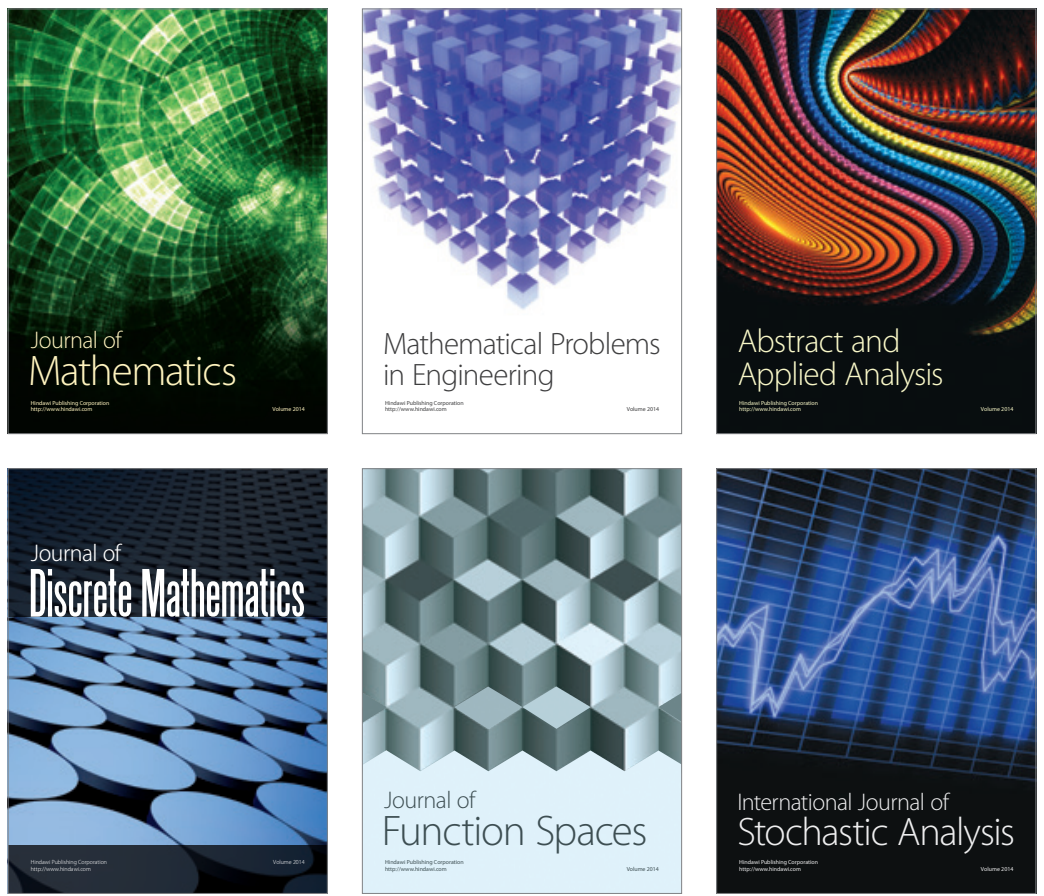

Journal of

Function Spaces

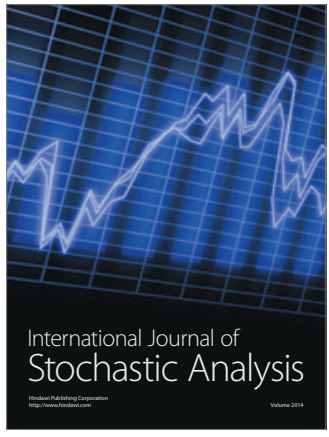

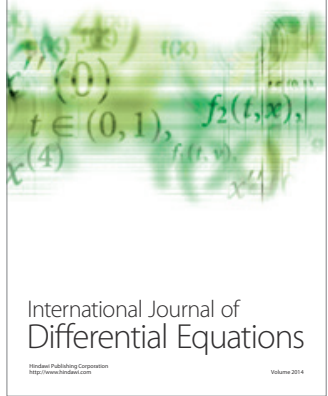
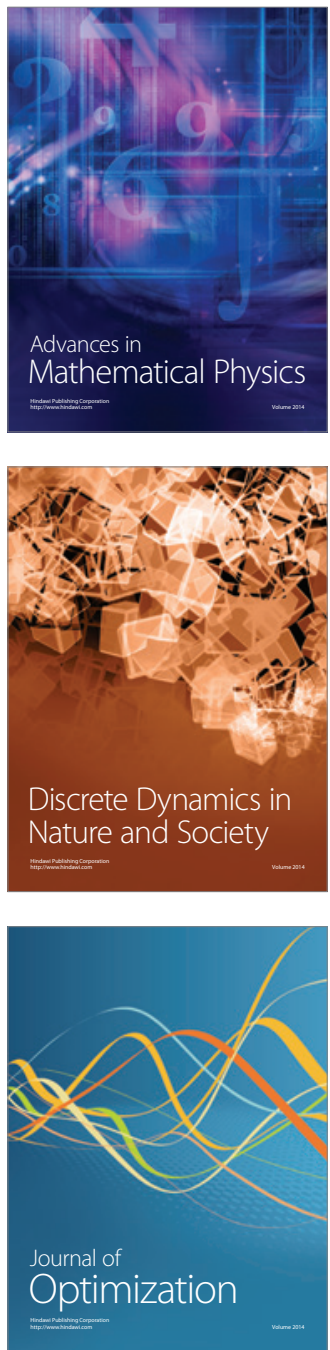